\title{
A retrospective study on adverse obstetric outcomes in HIV-infected pregnancy in West Bengal, India
}

\author{
Suman Ganguly ${ }^{1}$, Debjit Chakraborty ${ }^{2}$, Dipendra N. Goswami ${ }^{1,3}$ \\ ${ }^{1}$ West Bengal State AIDS Prevention and Control Society, India \\ ${ }^{2}$ State Urban Development Agency, West Bengal, India \\ ${ }^{3}$ Calcutta National Medical College, India
}

\begin{abstract}
Introduction: Human immunodeficiency virus (HIV) does not change the course of pregnancy, but seems to exert adverse obstetric outcomes like low birth weight, spontaneous abortion, or stillbirth. Antiretroviral treatment seems to reduce the chance of adverse outcomes. Frequently, HIV-infected women also seems to opt for medical termination of pregnancy (MTP). This study is to compare adverse obstetric outcomes and MTP rate for HIV-infected pregnancy with that in the general population irrespective of antiretroviral treatment (ART).

Material and methods: A retrospective cohort study was carried out through analysis of secondary data from 314 integrated counselling and testing centers across the state of West Bengal from October 2016 to December 2017. A total number of 227 HIV-infected on ART pregnancies, whose obstetric outcomes happened during the study period were followed up, and rate for low birth weight, spontaneous abortion, stillbirth, and MTP were calculated, and it was compared with still birth rate of the state among all pregnancies as per data of the District Level Household Survey 4 from 2013.

Results: Rates for low birth weight (28.04 per 100 pregnancy outcomes), spontaneous abortion (4.85 per 100), stillbirth (2.64 per 100), and MTP (9.25 per 100 live birth) were found to be significantly high for HIV-infected pregnancies on ART, as compared to the general population. MTP rates and low birth weight rates for general pregnancies did not fall within the $95 \%$ confidence interval of those rates for HIV-infected pregnancies.
\end{abstract}

Conclusion: HIV infection was responsible for significant adverse obstetric outcome irrespective of antiretroviral treatment.

HIV AIDS Rev 2020; 19, 1: 39-42

DOI: https://doi.org/10.5114/hivar.2020.93189

Key words: low birth weight, spontaneous abortion, still birth, MTP, HIV, ART.

\section{Introduction}

Prevention of parent-to-child transmission (PPTCT) basically aims at reducing the chance of transmission of human immunodeficiency virus (HIV) through vertical route. With

proper antiretroviral treatment (ART), the chance of transmission reduces significantly. Pregnancy does not seem to have an adverse effect on the natural history of HIV infection in women in most studies, although acquired immunodeficiency syndrome (AIDS) has become a leading cause

Article history:

Received: 05.08.2018

Received in revised form: 25.11 .2018

Accepted: 28.06.2019

Available online: 30.01 .2020
International Journal of HIV-Related Problems

HIV \& AIDS

R e v i e w 
of maternal mortality in some areas, as the epidemic progresses. Adverse pregnancy outcomes that have been reported in HIV-positive women include increased rates of spontaneous early abortion, low birth weight babies, and stillbirths, preterm labor, preterm rupture of membranes, other sexually transmitted diseases, bacterial pneumonia, urinary tract infections, and other infectious complications [1]. When taking reproductive decisions, the women living with HIV and AIDS take into consideration the fact they live with HIV and the possible risk of transmission of the virus to the fetus. Furthermore, they are subject to individual factors, social aspects, and cultural expectations. The literature on post-diagnosis abortion is quite limited [2], but indicates that the risk of transmission of the virus to the fetus was one of the factors that weighed on reproductive decisions. The introduction of the protocol for prevention of mother-to-child transmission (PMTCT) has produced a decrease in the rate of pregnancies ended in induced abortion [3].

In 2015 , there were 2.6 million stillbirths reported globally, with more than 7,178 daily deaths. The majority of these deaths occurred in developing countries. Congenital abnormalities, maternal complications, birth-related issues, fetal growth restrictions, maternal infections like malaria, syphilis, and HIV were responsible for adverse obstetric outcomes such as stillbirth [4].

So far, as Indian HIV epidemic scenario is concerned, the "India HIV Estimates 2015" shows that 2,116,581 people are living with HIV infection, with national prevalence of $0.26 \%$. Approximately, 86,309 new infections are reported yearly, and 35,255 HIV-infected pregnancies have been estimated to occur annually [5]. There is lack of studies in India related to the adverse pregnancy outcomes in HIV. No such study is reported from Eastern India. The aim of the study was to determine association of HIV-infected pregnancy with adverse obstetric outcomes. The specific objectives of the study were to compare adverse obstetric outcomes and MTP rate for HIV-infected pregnancy with that in the general population, irrespective of antiretroviral treatment.

\section{Material and methods}

This was retrospective record-based cohort study carried out through analysis of secondary data from October 2016 till December 2017. A total number of 227 HIV-infected on ART pregnant women whose obstetric outcomes happened during the study period were participating. A pre-designed format for tracking of individual details and obstetric outcome of positive pregnant women was used. Secondary data was collected as per designed format from PPTCT line list registers maintained in ICTCs. PPTCT line list is a standardized register for all facilities performing HIV confirmatory test especially for pregnant women. The information in this register is regularly updated, as the HIV-infected pregnant woman receives services along the cascades of PPTCT program. This updated line list data are shared with the state on monthly a basis in excel format and is monitored at the state level regarding completeness. However, information may not be available for lost to follow-up cases and those migrated to other states despite the best follow-up efforts; however, the number of such cases is very less to affect the study result. Operational case definition:

- still birth: a baby born without signs of life at/after 28 weeks' gestation,

- still birth rate: no. of stillbirth/all pregnancy outcomes $\times 100$,

- low birth weight: a baby born with birth weight less than 2,500 g,

- low birth weight rate: no. of babies with low birth weight/ all pregnancy outcomes $\times 100$,

- medical termination of pregnancy (MTP): an induced abortion performed in medical way for getting rid of unwanted pregnancy within $20^{\text {th }}$ week of gestation, completed by qualified medical practitioners trained in performing induced abortion,

- MTP rate: no. of pregnant women underwent MTP/all pregnancy outcomes $\times 100$,

- spontaneous abortion rate: no. of pregnant women underwent spontaneous abortion/all pregnancy outcomes $\times 100$.

Exclusion criteria included: 1) lost to follow-up cases, whose case record or obstetric outcomes are not known, however the number of such cases were very less (only 3), and 2) multiple pregnancies: only 1 such case was found and excluded; as multiple pregnancy per se, is perceived as a risk for adverse obstetric outcome, hence it may confound the estimated risk of HIV for adverse outcome of pregnancy.

To analyze the data, percentage of still birth rate, low birth weight rate, MTP rate, and spontaneous abortion rate were calculated for HIV-infected pregnancies for the study period using both the denominators i.e. with $(n=227)$ or without $(n=231)$ exclusion. 95\% confidence intervals (CIs) were calculated separately with two methods: 1) binomial exact calculation, and 2) normal approximation to the binomial calculation for both the rates, as obtained with or without exclusion of 4 cases in the denominator. This would exhibit the effect of using the exclusion on the study result, and the use of two methods for $95 \%$ confidence interval would increase the sensitivity as well as the precision for each subgroup (each type of adverse outcomes). This was compared with respective rates for all pregnancies (irrespective of HIV status), as available in the District Level Household and Facility Survey (DLHS-4) [6] data for the state (latest available data). The data was computed in MS Excel 2007 and presented as tables and line diagram. The data was further analyzed in Statistical Package for Social Science Software (version 17) to determine statistical significance for various attributes. For ethical consideration, shared confidentiality was maintained while collecting and analyzing the data. Each HIV screening was done with prior consent of the individual as per guideline of the National AIDS Control Organization.

\section{Results}

A total number of $231 \mathrm{HIV}$-infected pregnancies was registered in the PPTCT during the study period, out of which 
3 outcomes (1.3\%) were not known as they were lost to follow-up despite best efforts and $1(0.43 \%)$ was a multiple pregnancy. Rest $227 \mathrm{HIV}$-infected pregnancies, whose obstetric outcomes happened during the study period were considered for calculation of valid rate (\%). The rates for each adverse obstetric outcome using both 231 and 227 pregnancies as denominator is presented in Table 1 . Out of 227 pregnancies, adverse obstetric outcome was observed in 103 (45\%) pregnancies. Among them, 21 (9.21\%) opted for medical termination of pregnancy, 6 (2.64\%) resulted in stillbirth, 65 (28.63\%) ended with low birth weight babies, and 11 (4.85\%) resulted in spontaneous abortion. The rates for the same were $1.9 \%, 1 \%$, $11.5 \%$, and $2.5 \%$, respectively, among all pregnancies within the state as per DLHS-4 data (Table 2).

$95 \%$ confidence interval (CI) for all the rates, using two methods are presented in Table 2. The confidence interval did not include the respective rates in DLHS-4 data for MTP rates and low birth weight rates using both the methods. In case of spontaneous abortion, rates in DLHS-4 data was little higher than the lower limit of $95 \%$ confidence interval using both the method. In case of stillbirth rate, DLHS-4 figure corresponded with the lower limit of 95\% CI using binomial exact calculation (1\%), but was found to be little higher than the lower confidence limit when calculated by normal approximation method. However, numbers were much smaller for both spontaneous abor- tion and stillbirth (11 and 6, respectively). For all the rates, calculation using two denominators (227 and 231) was found to be similar in such a way that the ultimate inference in comparison to DLHS figures remained unaffected. However, we considered the rates obtained by using 227 as denominator valid one because keeping 231 pregnancies as denominator by default made to assume that no lost to follow-up case had any adverse outcome. This might lead to underestimation. Though keeping 227 as denominator might lead to little overestimation, 95\% confidence interval was precise and unaltered in regards to inference drawn. Since no maternal death was reported from this cohort; therefore, sensitivity analysis could not be carried out.

\section{Discussion}

In present study, stillbirth rate, rate for low birth weight and spontaneous abortion rate among the HIV-infected pregnancies were found to be as high as 2.60 (95\% CI: 1-5.6), 27.71 (95\% CI: 22.4-34.4), and 4.76 (95\% CI: 2.4-8.4), respectively as compared to $1,11.5$, and 2.5 , respectively, for all pregnancies in the state as per DLHS-4 data [6]. This was irrespective of ART status, as all the pregnant women recruited in this study were on ART. It was further noted that more number of HIV-infected pregnant women opted for medical termination of pregnancy despite being on ART as compared to the general population, though it was not statistically sig-

Table 1. Distribution of various adverse obstetric outcomes

\begin{tabular}{|c|c|c|c|}
\hline Obstetric outcomes & Number & $\begin{array}{c}\text { Rate (in \% unit per } \\
100 \text { pregnancy outcomes) } \\
(n=231)\end{array}$ & $\begin{array}{l}\text { Valid rate (in \% unit per } \\
100 \text { pregnancy outcomes) } \\
(n=227)\end{array}$ \\
\hline Medical termination of pregnancy & 21 & 9.09 & 9.25 \\
\hline Stillbirth & 6 & 2.60 & 2.64 \\
\hline Low birth weight & 65 & 27.71 & 28.63 \\
\hline Spontaneous abortion & 11 & 4.76 & 4.85 \\
\hline Total adverse obstetric outcome & 103 & 44.58 & 45.37 \\
\hline Total non-adverse outcome in single pregnancy & 124 & 53.68 & 54.63 \\
\hline Multiple pregnancy & 1 & 0.43 & \multirow{2}{*}{ Excluded from analysis } \\
\hline Lost to follow-up & 3 & 1.30 & \\
\hline
\end{tabular}

Table 2. Estimation of $95 \%$ confidence intervals for rates (\%) of adverse obstetric outcomes in HIV-infected pregnancies

\begin{tabular}{l|c|c|c|c|c|c|c|}
\hline $\begin{array}{l}\text { Adverse } \\
\text { obstetric } \\
\text { outcomes }\end{array}$ & $\begin{array}{c}\text { Rate } \\
(\%)\end{array}$ & $\begin{array}{c}\text { Valid rate } \\
(\%)\end{array}$ & \multicolumn{2}{|c|}{$\begin{array}{c}\text { 95\% confidence interval } \\
\text { of rate (\%) by binomial exact } \\
\text { calculation method }\end{array}$} & $\begin{array}{c}\text { 95\% confidence interval of rate } \\
\text { (\%) by normal approximation to } \\
\text { the binomial calculation method }\end{array}$ & $\begin{array}{c}\text { State DLHS-4 } \\
\text { data } \\
(\%)\end{array}$ \\
\cline { 4 - 7 } MTP rate & 9.09 & 9.25 & $5.7-13.6$ & $5.8-13.8$ & $5.4-12.8$ & $5.5-13.0$ & 1.9 \\
\hline Stillbirth rate & 2.60 & 2.64 & $1.0-5.6$ & $1.0-5.7$ & $0.5-4.6$ & $0.5-4.7$ & 1 \\
\hline $\begin{array}{l}\text { Low birth weight } \\
\text { rate }\end{array}$ & 27.71 & 28.63 & $22.4-34.4$ & $22.8-35.0$ & $21.9-33.5$ & $22.7-34.5$ & 11.5 \\
\hline $\begin{array}{l}\text { Spontaneous } \\
\text { abortion rate }\end{array}$ & 4.76 & 4.85 & $2.4-8.4$ & $2.4-8.5$ & $2.0-7.5$ & $2.0-7.6$ & 2.5 \\
\hline
\end{tabular}


nificant. This indicated the effectiveness of PMTCT counselling process with specific focus on Prong-2, which emphasized the management of unwanted pregnancies associated with HIV.

There are a lot of international studies demonstrating adverse obstetric outcomes of HIV-infected pregnancies. A study was conducted in Southwest Tshwane [7], which demonstrated that perinatal mortality rate and rate for low birth weight were higher in case of HIV-infected pregnancies, as compared to those who were non-infected. Our study corroborated these findings except the fact that spontaneous abortion rate was not found to be significantly higher in our study.

The association between HIV infection in pregnancy and obstetric outcomes is not uniformly established; there are several studies, which negate the fact that HIV infection in pregnancy does not necessarily increase the chance of adverse obstetric outcomes. One international study [8] says that as compared with no antiretroviral therapy or monotherapy, combination therapy for HIV-1 infection in pregnant women is not associated with increased rates of premature delivery, with low birth weight, low Apgar scores, or stillbirth in their infants. Our study population were using combination antiretroviral treatment and in spite of that, results showed increased stillbirth rate and spontaneous abortion in HIV-infected pregnancy. As per another Sub-Saharan Africa-based study, HIV status of a pregnant woman was not directly linked to probability of stillborn infant [9]. This finding needs further validation.

Our study is unique, as limited number of similar studies has been conducted in India, and there is lack of similar studies in Eastern India and low prevalence setting. One study from Bangalkote district [10] in Karnataka state showed high level of pregnancy loss in HIV-infected pregnancies, but could not demonstrate any association between low birth weight, MTP rate, and HIV-infected pregnancies. The study did not include the effect of ART.

Regarding opting for medical termination of pregnancy in HIV-infected pregnant women, it is comparatively high as compared to the HIV-negative counterparts. There are very few number of studies in this regards. An Italy-based original research suggested that HIV diagnosis was predictive of abortion only in the 1980s [11]. Similarly, high prevalence of unwanted pregnancy and induced abortion was demonstrated in Western India-based study [12].

\section{Conclusions}

HIV infection can cause adverse pregnancy outcome irrespective of ART status and increased rate of MTP for unwanted pregnancies is acceptable under such circumstances. Molecular level studies are needed to corroborate the fact further.

\section{Limitation}

The current study has several limitations. 1. We did not find any ART naive mother as a comparison group. 2. Com- paring the rates of 2016-17 with the DLHS figure of 20122013 may not be ideal, however in absence of recent state figure of adverse obstetric outcome of all pregnancies, DLHS-4 data was used for comparison. 3. The outcome of 3 cases could not be traced despite the best efforts to follow-up. However, the denominator had to be reduced for calculation of valid rates, since we did not have any previous rates from the state for HIV-infected pregnancy to estimate and censor based on that. We used two different method, while calculating 95\% confidence interval, and the result was not affected as number of lost to follow-up cases was very small. 4 . Since no maternal death was reported from this cohort of HIV-infected pregnancies, the comparison of maternal mortality rates and sensitivity analysis were not carried out. No censoring for lost to follow-up cases were done, since these cases did not differ from other cases in terms of pregnancy complications.

\section{Conflict of interest}

The authors declare no conflict of interest with respect to the research, authorship, and/or publication of this article.

\section{References}

1. World Health Organization and Joint United Nations Programme on HIV/AIDS (UNAIDS), 1998. HIV in pregnancy: a review. Available at: http://www.unaids.org/sites/default/files/media_asset/ jc151-hiv-in-pregnancy_en_1.pdf [Accessed: 23.02.2017].

2. Pilecco FB. Aborto Provocado em Mulheres Vivendo com HIV/AIDS, Porto Alegre, Universidade Federal do Rio Grande do Sul, 2014. Available at: https://scholar.google.com.br [Accessed: 04.08.2018].

3. Pilecco FB, Teixeira LB, Vigo A, Knauth DR. Post-diagnosis abortion in women living with HIV/AIDS in the south of Brazil. Ciência \& Saúde Coletiva 2015; 20: 1521-1530.

4. WHO updates - Maternal, newborn, child and adolescent health - Stillbirths. Available at: http://www.who.int/maternal_child_adolescent/epidemiology/stillbirth/en/ [Accessed: 23.02.2017].

5. India HIV Estimation 2015, Technical Report. National AIDS Control Organisation \& National Institute of Medical Statistics, ICMR, Ministry of Health \& Family Welfare. Government of India.

6. District Level Household and Facility Survey-4: State Fact Sheet West Bengal 2012-13. Available at: rchiips.org/pdf/dlhs4/report/ WB.pdf [Accessed: 06.02.2018].

7. Pattinson RC, Hulsbergen MH, Van Hoorick L. The effect of maternal HIV infection on maternal conditions and perinatal deaths in southwest Tshwane. Facts Views Vis Obgyn 2010; 2: 227-231.

8. Kim HY, Kasonde P, Mwiya M, et al. Pregnancy loss and role of infant HIV status on perinatal mortality among HIV-infected women. BMC Pediatrics 2012; 12: 138.

9. Ball H. HIV may not increase risk of stillbirth among women in Sub-Saharan Africa. International Perspectives on Sexual And Reproductive Health 2008; 34: 53.

10. Halli SS, Khan CG, Shah I, et al. Pregnancy wastage among HIV infected women in a high HIV prevalence district of India. BMC Public Health 2015; 15: 602.

11. Ammassari A, Cicconi P, Ladisa N, et al. Induced first abortion rates before and after HIV diagnosis: results of an Italian self-administered questionnaire survey carried out in 585 women living with HIV. HIV Med 2013; 14: 31-39.

12. Darak S, Hutter I, Kulkarni V, Kulkarni S, Janssen F. High prevalence of unwanted pregnancies and induced abortions among HIV-infected women from Western India: need to emphasize dual method use? AIDS Care 2016; 28: 43-51. 